\title{
Kosten-Nutzen-Bewertung (HTA) von medizinischen Leistungen und Heilmitteln
}

Dr. rer. pol. Heinz Locher

Berater im Gesundheitswesen, Bern

Podiumsgäste

- Dr. phil. nat. Bernhard Wegmüller, Direktor H+ Die Spitäler der Schweiz

- Dr. med. Markus Trutmann, Generalsekretär fmCh

- PD Dr. Simon Hölzer, Geschäftsführer SwissDRG AG

- Dr. med. Pierre-François Cuénoud, Mitglied des Zentralvorstandes der FMH

- Dr. rer. pol. Melchior Buchs, Generalsekretär FASMED

Korrespondenz:

Roswitha Scheidweiler

RS Medical Consult GmbH

Frauenberg 1

D-97980 Bad Mergentheim

Tel. +49793152612

Fax +497931561226

info@rsmedicalconsult.com

www.rsmedicalconsult.com
Die Fallpauschalen kommen. So hat es der Gesetzgeber entschieden. Soviel ist sicher. Welche Auswirkungen das neue Tarifsystem auf die medizinische Versorgung der Bevölkerung haben wird, ist viel weniger sicher. Werden die Gesundheitskosten sinken oder noch stärker ansteigen als bisher? Kann das hohe Qualitätsniveau der Schweizer Medizin gerettet werden? Werden innovative Pharma- und Medtech-Produkte bezahlbar sein? Mit diesen und anderen gesundheitsökonomischen Fragen muss sich die Begleitforschung befassen.

Deutschland hat es lange Zeit versäumt, eine solche Begleitforschung zu betreiben. Diesen Fehler sollte die Schweiz nicht wiederholen.

Ob der laufenden Diskussionen im Hinblick auf die Einführung der SwissDRG darf nicht vergessen gehen, dass weitere Reformen erforderlich sind. Diese sollten vor allem der dringend erforderlichen Verbesserung des Kosten-Nutzen-Verhältnisses unseres Gesundheitswesens dienen. Verschiedene der anstehen- den Fragen sind auch in vergleichbaren ausländischen Gesundheitssystemen aktuell. Deshalb drängt es sich auf, durch Vermittlung renommierter Referenten von ausländischen Lösungen, Erfahrungen und Schlussfolgerungen zu lernen. Vor dem Hintergrund der Ausführungen zweier renommierter internationaler Experten erläutern an dieser hochkarätigen Tagung in der Folge Exponenten der wichtigsten Partner des schweizerischen Gesundheitswesens aus ihrer je speziellen Optik ihre Erwartungen und Prioritäten. Daraus soll sich ein Aktionsplan für die nächsten Reformschritte ergeben.

Das Symposium richtet sich an Verantwortungsträger und Akteure aus allen Bereichen des schweizerischen Gesundheitswesens: Krankenversicherer, Spitäler, Behördenvertreter von Bund und Kantonen, Pharma- und Medizinaltechnikindustrie; Universitätsforscher; Verbände des Gesundheitswesens; am Gesundheitswesen interessierte Politikerinnen und Politiker und Medienschaffende.

\section{Aktuelle Herausforderungen des schweizerischen Gesundheitswesens: Schlussfolgerungen und Empfehlungen aus internationaler Sicht}

Programm: 17. Juni 2009, Zürich, 9.00-17.00 Uhr

8.30 Uhr Empfang bei Kaffee und Teea

9.00 Uhr Begrüssung durch Dipl.-Kauffrau Roswitha Scheidweiler, RS Medical Consult GmbH

9.05 Uhr Dr. iur. Carlo Conti: Was erwarten wir von der Einführung der DRGs in der Schweiz? z.B. zum Thema Begleitforschung

9.25 Uhr Dr. med. Peter Indra MPH: Was erwarten Bund und Politik von der Einführung der DRGs in der Schweiz?

9.45 Uhr Prof. Dr. med. Michael Heberer: Begleitforschung: Erwartungen und Beiträge der Spitäler

\section{$10.05 \mathrm{Uhr}$ Kaffeepause}

10.30 Uhr Dr. med. Daniel Herren: Wünsche an die Begleitforschung aus der Sicht der Ärzteschaft

10.50 Uhr Pius Gyger, lic. oec.: Sicht der Krankenversicherung

11.15 Uhr Prof. Dr. Reinhard Busse: Internationale Gesundheitssystementwicklungen und daraus resultierende Überlegungen für das schweizerische Gesundheitswesen

12.00 Uhr Diskussions- und Fragerunde mit den Podiumsgästen

13.00 Uhr Gemeinsames Mittagessen

14.00 Uhr Prof. Dr. Uwe Siebert: HTA-Methoden; Kosten-Nutzen-Bewertung; Entscheidungsanalysen und die Bewertung von Arzneimitteln aus internationaler Perspektive

14.45 Uhr Dr. Ansgar Hebborn: International eingesetzte Verfahren der Kosten-Nutzen-Bewertung von Medikamenten und anderer Gesundheitstechnologien sowie resultierende Überlegungen für die Schweiz aus der Perspektive der forschenden Arzneimittelindustrie

15.05 Uhr Kaffeepause

15.30 Uhr Margit Kessler: Kosten-Nutzen-Bewertung aus Patientensicht

15.50 Uhr Diskussions- und Fragerunde mit den Podiumsgästen

16.50 Uhr Schlusswort

17.00 Uhr Gedankenaustausch unter den Teilnehmenden mit Schlussaperitif. 